\title{
Upper Bounds for the Energy Expectation in Time-Dependent Quantum Mechanics
}

\author{
Alain Joye $e^{1,2}$
}

\author{
Received November 13, 1995
}

We consider quantum systems driven by Hamiltonians of the form $H+W(t)$, where the spectrum of $H$ consists of an infinite set of bands and $W(t)$ depends arbitrarily on time. Let $\langle H\rangle_{\varphi}(t)$ denote the expectation value of $H$ with respect to the evolution at time $t$ of an initial state $\varphi$. We prove upper bounds of the type $\langle H\rangle_{\varphi}(t)=O\left(t^{\delta}\right), \delta>0$, under conditions on the strength of $W(t)$ with respect to $H$. Neither growth of the gaps between the bands nor smoothness of $W(t)$ is required. Similar estimates are shown for the expectation value of functions of $H$. Sufficient conditions to have uniformly bounded expectation values are made explicit and the consequences on other approaches to quantum stability are discussed.

KEY WORDS: Quantum stability; energy expectations; quantum diffusion.

\section{INTRODUCTION}

Consider a time-dependent system characterized by a Hamiltonian of the form

$$
H+W(t)
$$

where $H$ is a positive self-adjoint operator whose spectrum consists of separated bands $\left\{\sigma_{j}\right\}_{j=1}^{\infty}$ such that

$$
\sigma_{j} \subseteq\left[\lambda_{j}, A_{j}\right]
$$

\footnotetext{
' Department of Mathematics and Center for Transport Theory and Mathematical Physics, Virginia Polytechnic Institute and State University, Blacksburg, Virginia 24061-0123.

2 Permanent address: Centre de Physique Théorique, CNRS Marseille, Luminy Case 907 , F-13288 Marseille Cedex 9, France, and PHYMAT, Université de Toulon et du Var, B.P. 132, F-83957 La Garde Cedex, France.
} 
and $W(t)$ is a time-dependent symmetric perturbation. Let $U(t)$ be the corresponding evolution operator satisfying the Schrödinger equation

$$
i U^{\prime}(t) \varphi=(H+W(t)) U(t) \varphi, \quad U(0)=\mathbf{I}
$$

where the prime denotes time derivative.

Our main concern is the time behavior as $t \rightarrow \infty$ of the expectation value of the energy operator $H$,

$$
\langle H\rangle_{\varphi}(t)=\langle U(t) \varphi \mid H U(t) \varphi\rangle
$$

and of similar operators. These are among the quantities of interest in the study of quantum stability for general time-dependent systems; see, e.g., refs. $5,1,11,13$, and 19 and references therein. Such quantities have been studied analytically for driven quantum oscillators ${ }^{(5.7 .1)}$ for various time dependences. The solubility of the quantum problem and its strong links with the classical dynamics of the system make it possible to get a rather precise description of the expectation value of the kinetic energy. For example, it can be deduced from the analysis provided in ref. 7 that periodically perturbed harmonic oscillators can lead to a behavior of the type

$$
\langle H\rangle_{\varphi}(t) \simeq e^{\gamma t}, \quad \gamma>0
$$

for some parameters and some initial condition $\varphi$ (G. Hagedorn, private communication).

For general Hamiltonians of the form (1.1) with arbitrary time dependence, the only analytical results we are aware of are those of Nenciu, ${ }^{(18)}$ who tackles this problem by means of tools coming from the adiabatic theory. The adiabatic machinery already proved to be useful in the determination of the spectral properties of the monodromy operator in case of periodic time dependence of the Hamiltonian; see, e.g., refs. 8, 9, 17, $12,18,3$, and 4 . Nenciu considers systems with increasing gaps in ref. 18 and the main result regarding $\langle H\rangle_{\varphi}(t)$ is essentially that if the gaps $\lambda_{j}-\Lambda_{j-1}$ between the bands grow like $j^{\alpha}$, with $\alpha>0$, and if $W(t)$ is strongly $C^{\prime \prime}$ with $n \geqslant[(1+\alpha) /(2 \alpha)]+1$, then

$$
\langle H\rangle_{\varphi}(t)=O\left(t^{(1+\alpha) / n \alpha}\right)
$$

as $t \rightarrow \infty$, provided sup $r \in \mathbf{R}^{+}\left\|(d / d t)^{k} W(t)\right\|<\infty, k=0,1, \ldots, n$. This estimate holds for arbitrary time dependence of $W(t)$ and regardless of the nature of the spectrum in the bands $\sigma_{j}$. The length of the bands must not grow faster than $j^{\alpha}$. Note that the necessary growth of the gaps in the spectrum of $H$ prevents the application of this result to the driven harmonic oscillator. 
In this paper we also deal with $H$ 's whose spectrum consists of an infinite set of bands and we obtain results which can be considered as complementary to those of $\mathrm{Nenciu}^{(18)}$ in the following sense. We prove estimates similar to (1.6), without restriction on the size of gaps and without a smoothness assumption on $W(t)$. Of course, there is a price to pay for dropping these hypotheses: the strength of perturbation $W(t)$ with respect to $H$ must be small in some sense. Let us denote the spectral projectors of $H$ associated with the band $\sigma_{j}$ by $P_{j}$. Typically, if the operator $W(t)$ is such that

$$
\sup _{s \in \mathbf{R}^{+}} \sum_{j=1}^{\infty} \lambda_{j}^{2 q}\left\|P_{j} W(s)\right\|^{2}<\infty
$$

for some $q \geqslant 1 / 2$, then, as $t \rightarrow \infty$,

$$
\langle H\rangle_{\varphi}(t)=O\left(t^{1 / q}\right)
$$

See Section 2. Here again, (1.8) holds for arbitrary time dependence of $W(t)$ and regardless of the nature of the spectrum in the bands $\sigma_{j}$ provided the bands are not too long. Such algebraic bounds on the growth of $\langle H\rangle_{\varphi}(t)$ already give some information on the system; see (1.5). However, as noticed in ref. 18, for $C^{1}$ perturbations such that both $\|W(t)\|$ and $\left\|W^{\prime}(t)\right\|$ are uniformly bounded in time we have the trivial bound for any $H$

$$
\langle H\rangle_{\varphi}(t)=O(t)
$$

If we work under stronger hypotheses, more precise estimates are given, some of which lead to uniformly bounded expectation values. This is the case if we assume instead of (1.7) that the function of $s$ defined by

$$
\left(\sum_{j=1}^{\infty} \lambda_{j}\left\|P_{j} W(s)\right\|^{2}\right)^{1 / 2}
$$

is integrable on $\mathbf{R}^{+}$.

Remarking that (1.8) is independent of the characteristics of the bands in the spectrum, we can generalize our results to estimate the expectation values of reasonable positive functions of $H, f(H)$, in the following way (see Section 3). Assume for simplicity that $f: \mathbf{R}^{+} \rightarrow \mathbf{R}^{+}$is strictly increasing and that (1.7) is satisfied with $f\left(\lambda_{j}\right)$ in place of $\lambda_{j}$. Then

$$
\langle f(H)\rangle_{\varphi}(t)=O\left(t^{1 / q}\right)
$$


Also, if (1.10) is satisfied with $f\left(\lambda_{j}\right)$ in place of $\lambda_{j}$, $\sup _{t \in \mathbf{R}^{+}}\langle f(H)\rangle_{\varphi}(t)<\infty$. Thus it may be possible to have $\sup _{t \in \mathbf{R}^{+}}\langle f(H)\rangle_{\varphi}(t)<\infty$ for a careful choice of $f$, whereas $\langle H\rangle_{\varphi}(t)$ cannot be uniformly bounded by our methods. A specific example displaying these features is dealt with in Section 4. Such cases are of interest when we consider other relevant quantities in the study of quantum stability; see Section 6.

In Section 5 we consider Hamiltonians $H$ with discrete spectrum such that $\lambda_{j} \simeq j^{\alpha}, \alpha>0$. In particular, we show that perturbations $W(t)$ belonging to a class of operators introduced by Howland ${ }^{(10)}$ satisfy our hypotheses. This class is determined by the behavior of the matrix elements of $W(t)$ in a basis of eigenvectors of $H$ and was considered by Howland in his study of the spectral properties of the monodromy operator for timeperiodic $W(t)$. We end the paper by addressing some questions on the links between results on expectation values of operators and different linear manifolds introduced to study the quantum stability of such systems in Section 6.

The method used to get our estimates essentially consists in comparing the actual evolution $U(t)$ with the free evolution $V(t)=e^{-i t /}$ and controlling the difference between them. In some sense, this can be viewed as some kind of degenerate adiabatic technique. Therefore, our proof follows the general pattern of that of Nenciu. ${ }^{(18)}$ By slightly modifying the method sketched above, we can actually accommodate a certain class of unbounded perturbations $W(t)$, for which (1.9) does not apply.

Before closing this introduction, let us note that the search for lower bounds on $\langle f(H)\rangle_{\varphi}(t)$ is more complicated. This is due in particular to the fact that such bounds must depend on the initial state $\varphi$, whereas this is not the case for upper bounds. There are results for time-independent systems only which require knowledge of the detailed structure of the spectral measure associated with $\varphi$. The interested reader should consult refs. 6,2 , and 16 and references therein.

\section{UPPER BOUNDS ON $\langle H\rangle_{\varphi}(t)$}

We consider a Hamiltonian $H$ satisfying the following requirements.

Hypothesis H1. $H$ is a positive self-adjoint operator with dense domain $D(H)$ in a separable Hilbert space $\mathscr{H}$. The spectrum $\sigma$ of $H$ consists of disjoint bands $\sigma=\left\{\sigma_{j}\right\}_{j=1}^{\infty}$ characterized by

$$
\lambda_{j}=\inf _{\lambda \in \sigma_{j}} \lambda \quad \text { and } \quad \Lambda_{j}=\sup _{\lambda \in \sigma_{j}} \lambda
$$


with $\Lambda_{j}<\lambda_{j+1} \forall j \in \mathbf{N}^{*}=\{1,2, \ldots\}$. Moreover,

$$
\lim _{j \rightarrow \infty} \lambda_{j}=\infty \quad \text { and } \quad \sup _{j \in N^{*}} \Lambda_{j} / \lambda_{j}=s<\infty
$$

Let us denote by $P_{j}$ the spectral projection corresponding to $\sigma_{j}, j \in \mathbf{N}^{*}$, by means of the Riesz formula. We set

$$
m_{j}=\operatorname{dim} \operatorname{Ran}\left(P_{j}\right) \leqslant \infty
$$

allowing infinite values of $m_{j}$.

The time-dependent perturbation $W(t)$ is such that:

Hypothesis $\mathbf{H 2}$. $W(t), t \in \mathbf{R}^{+}$, is a symmetric, strongly $C^{1}$ operator which is $H$-bounded,

$$
\|W(t) \varphi\| \leqslant a(t)\|\varphi\|+b\|H \varphi\|
$$

where $b<1$ is uniform in $t$ and $a(t)<\infty \forall t \in \mathbf{R}^{+}$. The diagonal part of $W(t)$ is defined by the strong limit

$$
W^{d}(t) \varphi=\sum_{j=1}^{\infty} P_{j} W(t) P_{j} \varphi, \quad \varphi \in D(H)
$$

and we further assume that the off-diagonal part of $W(t)$,

$$
W^{o}(t)=W(t)-W^{d}(t)
$$

is bounded. Moreover, there exists a subsequence going to infinity $\left\{j_{k}\right\}_{k=1}^{\infty} \subset \mathbf{N}^{*}$ such that $\left\|P_{j k} W^{o}(t)\right\| \not \equiv 0$.

Remark. This last assumption ensures that subspaces of arbitrary energy are coupled by $W^{o}(t)$. Otherwise, $\langle H\rangle_{\varphi}(t)$ is uniformly bounded in time.

It follows from $\mathrm{H} 1$ and $\mathrm{H} 2$ that the operator

$$
H(t)=H+W(t)
$$

is strongly $C^{1}$, self-adjoint on $D(H)$, and bounded from below for all $t \in \mathbf{R}^{+}$(ref. 14, §V.4). Thus the evolution operator $U(t)$ satisfying the Schrödinger equation

$$
i U^{\prime}(t) \varphi=(H+W(t)) U(t) \varphi, \quad U(0)=\mathbf{I}
$$

where $\varphi \in D(H)$ and $t \in \mathbf{R}^{+}$is unitary and both $U(t), U^{-1}(t)$ map $D(H)$ into $D(H)$ (see, e.g., ref. 15). 
We now approximate the evolution $U(t)$ by a unitary $V(t)$ which is diagonal with respect to $\left\{P_{j}\right\}_{j=1}^{\infty}$ and such that the difference $U(t)-V(t)$ is small in some sense.

Lemma 2.1. The operator $W^{d}(t)$ is symmetric, $H$-bounded with relative bound $b$ for any $t \in \mathbf{R}^{+}$, and is strongly $C^{1}$.

Remark. As a consequence, the bounded operator $W^{o}(t)$ is strongly $C^{1}$ and self-adjoint.

It follows from this lemma, the proof of which is given in an appendix, that the solution of

$$
i V^{\prime}(t) \varphi=\left(H+W^{d}(t)\right) V(t) \varphi, \quad V(0)=\mathbf{I}
$$

where $\varphi \in D(H)$ and $t \in \mathbf{R}^{+}$, is unitary and both $V(t), V^{-1}(t)$ map $D(H)$ into $D(H)$.

Moreover, as a consequence of

$$
\left(H+W^{d}(t)\right) P_{j} \varphi=P_{j}\left(H+W^{d}(t)\right) \varphi, \quad \forall j \in \mathbf{N}^{*}, \quad t \in \mathbf{R}^{+}, \quad \varphi \in D(H)
$$

we have the identity

$$
\left[V(t), P_{j}\right] \equiv 0, \quad \forall j \in \mathbf{N}^{*}, \quad t \in \mathbf{R}^{+}
$$

The above properties yield

$$
\begin{aligned}
& i\left(V^{-1}(t) U(t)\right)^{\prime} \varphi \\
& \quad=i V^{-1}(t) U(t) \varphi+i V^{-1}(t) U(t)^{\prime} \varphi \\
& \quad=V^{-1}(t)\left(W(t)-W^{d}(t)\right) U(t) \varphi=V^{-1}(t) W^{o}(t) U(t) \varphi
\end{aligned}
$$

for any $\varphi \in D(H)$, where $W^{o}(t)$ is assumed to be bounded. Or, in an equivalent form,

$$
U(t)-V(t)=-i V(t) \int_{0}^{t} V^{-1}(s) W^{o}(s) U(s) d s \equiv R(t)
$$

where the integral is taken in the strong sense and

$$
\|R(t)\| \leqslant 2
$$

Note that $R(t)$ maps $D(H)$ into $D(H)$ since $U(t), V(t)$, and $V^{-1}(t)$ do. However, we will be able to take initial conditions in the form domain $Q$ 
of $H$, i.e., in $Q=D\left(H^{1 / 2}\right)$ (ref. 14 , §VI.2.6). For any $\chi, \varphi \in D\left(H^{1 / 2}\right)$, we will use the notation

$$
\langle\chi \mid H \varphi\rangle=\left\langle H^{1 / 2} \chi \mid H^{1 / 2} \varphi\right\rangle
$$

Let us finally introduce $H$-dependent norms on the space of bounded operators which will appear in the estimates to come.

Definition. The $H$-norm of order $p \geqslant 1$ of a bounded operator $B$ is defined by

$$
\|B\|_{p . H}=\left(\sum_{j=1}^{\infty} \lambda_{j}^{p}\left\|P_{j} B\right\|^{p}\right)^{1 / p}
$$

where $\lambda_{j}$ and $P_{j}$ are given in $\mathrm{Hl}$.

It is easily checked that $\|B\|_{p, H}$ is indeed a norm, using Minkowski's inequality. When $p=2,\|B\|_{2 . H}$ is related to the usual norm and HilbertSchmidt norm (denoted by $\|\cdot\|_{2}$ ) of $H B$ in the following way.

Lemma 2.2. With $s, m_{j}$ defined by (2.2), (2.3), respectively and $\sup _{j \in \mathrm{N}^{*}} m_{j}=M \leqslant \infty$, we have

$$
\|H B\| \leqslant s\|B\|_{2, H} \leqslant s\|H B\|_{2} \leqslant \sqrt{M} s^{2}\|B\|_{2, H}
$$

If $m_{j}=1 \forall j \in \mathbf{N}^{*}$, we have $s=M=1$ and equal signs in place of the last two inequalities.

The proof is given in an appendix.

To control the size of $R(t)$ we must make assumptions on the size of $W^{o}(t)$. We consider a set of two hypotheses giving rise to different results:

Hypothesis H3. (i) There exists $q \geqslant 1 / 2$ such that

$$
\left(\sum_{j=1}^{\infty} \lambda_{j}^{2 q}\left\|P_{j} W^{o}(t)\right\|^{2}\right)^{1 / 2} \equiv\left\|W^{o}(t)\right\|_{2, H^{q}}
$$

is locally integrable as a function of $t$.

(ii) Hypothesis $\mathrm{H} 3(\mathrm{i})$ holds and $W^{o}(t)$ has the form

$$
W^{o}(t)=k(t) \omega^{o}
$$

where $k(t)$ is a real-valued function and $\omega^{\circ}$ is a bounded, self-adjoint, timeindependent operator on $\mathscr{H}$. 
Note that the $P_{j}$ and $\lambda_{j}^{q}$ are the spectral projectors and bottoms of the bands of $H^{q}$, respectively, so that the norms introduced here are consistent with our definition above.

Theorem 2.1. Assume hypotheses $\mathrm{H} 1-\mathrm{H} 3$. Then the evolution operator solution of (2.8) is such that for any $t \in \mathbf{R}^{+}$

$$
U(t): \quad D\left(H^{1 / 2}\right) \rightarrow D\left(H^{1 / 2}\right)
$$

Let $\varphi \in D\left(H^{1 / 2}\right)$ and consider $\langle H\rangle_{\varphi}(t)=\langle U(t) \varphi \mid H U(t) \varphi\rangle$ as $t \rightarrow \infty$. If $\mathrm{H} 3(\mathrm{i})$ holds, then

$$
\langle H\rangle_{\varphi}(t)=O\left(\left[\int_{0}^{t}\left\|W^{o}(s)\right\|_{2, H^{q}} d s\right]^{1 / q}\right)
$$

If, in addition, $\sum_{j=1}^{\infty} \lambda_{j}^{2 q}\left\|P_{j} W^{o}(s)\right\|^{2}$ converges uniformly in $s \in \mathbf{R}^{+}$, we get

$$
\langle H\rangle_{\varphi}(t)=o\left(t^{1 / q}\right)
$$

If $\mathrm{H} 3$ (ii) holds, then

$$
\langle H\rangle_{\varphi}(t)=o\left(\left[\int_{0}^{t}|k(s)| d s\right]^{1 / q}\right)
$$

Remarks. 1. No assumptions are made on the nature of the spectrum of $H$ inside the bands $\sigma_{j}$, the size of the gaps between them, or on the actual dependence of $\lambda_{j}$ on $j$. The time dependence of $W(t)$ is restricted in no way and the norm or $H$-norm of $W^{o}(t)$ need not be uniformly bounded in time.

2. The initial condition can be taken in $D\left(H^{1 / 2}\right) \supset D(H)$.

3. If $\mathrm{H} 3(\mathrm{i})$, respectively $\mathrm{H} 3(\mathrm{ii})$, hold and $\left\|W^{o}(s)\right\|_{2, H^{4}}$, respectively $k(s)$, are integrable, we thus get $\sup _{t \in \mathbf{R}^{+}}\langle H\rangle_{\varphi}(t)<\infty$. This could have been expected in the latter case since $\int_{0}^{\infty}|k(s)| d s$ is a measure of the total amount of energy available to the system.

4. If $\left\|W^{o}(s)\right\|_{2 . H^{4}}$ is uniformly bounded as a function of time, we get the estimate

$$
\langle H\rangle_{\varphi}(t)=O\left(t^{1 / q}\right)
$$

5. The additional result (2.22) holds, for example, under the stronger assumption on $W^{\circ}(s)$

$$
\sum_{j \in N^{+}} \lambda_{j}^{2 g} \sup _{t \in \mathbf{R}^{+}}\left\|P_{j} W^{o}(t)\right\|^{2}<\infty
$$


6. The bound (2.24) when $q \in[1 / 2,1]$ is weaker than the trivial bound (1.9) for bounded $W(t)$, but it is of interest if $W(t)$ is unbounded.

7. The unitary $V(t)$ defined by (2.9) could be replaced by $e^{-i t H}$ when $W(t)$ is bounded, but in hypothesis $\mathrm{H} 3, W^{o}(t)$ should be replaced by $W(t)$, which implies stronger conditions to fulfill. However, such a choice makes it possible to accommodate bounded $W(t)$ which are not $C^{1}$ but generate a well-behaved evolution operator.

8. The parameter $q$ characterizes the relative strength of the perturbation $W(t)$ with respect to $H$. The higher $q$ is, the weaker is $W(t)$ and the weaker is the growth in time of energy.

Proof. A consequence of hypothesis H3(i) is that for any $t \in \mathbf{R}^{+}$and any $q \geqslant 1 / 2$

$$
\begin{aligned}
\sum_{j=1}^{\infty}\left(\int_{0}^{l} \lambda_{j}^{q}\left\|P_{j} W^{o}(s)\right\| d s\right)^{2} & \leqslant\left[\int_{0}^{1}\left(\sum_{j=1}^{\infty} \lambda_{j}^{2 q}\left\|P_{j} W^{o}(s)\right\|^{2}\right)^{1 / 2} d s\right]^{2} \\
& \equiv\left(\int_{0}^{1}\left\|W^{o}(s)\right\|_{2, H^{q}} d s\right)^{2}
\end{aligned}
$$

Indeed, the partial sums depending on two variables $(s, u) \in[0, t] \times[0, t]$ satisfy

$$
\begin{aligned}
& \sum_{j=1}^{N} \lambda_{j}^{q}\left\|P_{j} W^{o}(s)\right\| \lambda_{j}^{q}\left\|P_{j} W^{o}(u)\right\| \\
& \quad \leqslant\left(\sum_{j=1}^{N} \lambda_{j}^{2 q}\left\|P_{j} W^{o}(s)\right\|^{2}\right)^{1 / 2}\left(\sum_{j=1}^{N} \lambda_{j}^{2 q}\left\|P_{j} W^{o}(u)\right\|^{2}\right)^{1 / 2} \\
& \quad \leqslant\left\|W^{o}(s)\right\|_{2, H^{q}}\left\|W^{o}(u)\right\|_{2 . H^{4}}
\end{aligned}
$$

where the right member is integrable on $[0, t] \times[0, t]$ by assumption for any $t \in \mathbf{R}^{+}$. Hence we get the result by integration on $(s, u) \in[0, t] \times[0, t]$ from the dominated convergence theorem.

On the other hand, we get from the hypotheses made on $H$

$$
H=\sum_{j=1}^{\infty} H_{j}=\sum_{j=1}^{\infty} \int_{\lambda_{j}}^{A_{j}} \lambda d E(\lambda)
$$

where $E(\lambda)$ is the spectral family of $H$ and $H_{j}=P_{j} H P_{j}$ satisfies for any $j \in \mathbf{N}^{*}$

$$
\lambda_{j} P_{j} \leqslant H_{j} \leqslant \Lambda_{j} P_{j} \leqslant s \lambda_{j} P_{j}
$$


[see (2.2)]. Also

$$
D\left(H^{1 / 2}\right)=\left\{\varphi \in \mathscr{H} \mid \sum_{j=1}^{\infty} \int_{\lambda_{j}}^{A_{j}} \lambda d\|E(\lambda) \varphi\|^{2}<\infty\right\}
$$

But since

$$
\lambda_{j}\left\|P_{j} \varphi\right\|^{2} \leqslant \int_{\lambda_{j}}^{\lambda_{j}} \lambda d\|E(\lambda) \varphi\|^{2} \leqslant s \lambda_{j}\left\|P_{j} \varphi\right\|^{2}
$$

we get

$$
D\left(H^{1 / 2}\right)=\left\{\varphi \in \mathscr{H} \mid \sum_{j=1}^{\infty} \lambda_{j}\left\|P_{j} \varphi\right\|^{2}<\infty\right\}
$$

Take $\varphi \in D\left(H^{1 / 2}\right)$. Using (2.11), we get

$$
\sum_{j=1}^{\infty} \lambda_{j}\left\|P_{j} V(t) \varphi\right\|^{2}=\sum_{j=1}^{\infty} \lambda_{j}\left\|P_{j} \varphi\right\|^{2}<\infty
$$

so that $V(t) \varphi \in D\left(H^{1 / 2}\right)$. Consider now $R(t) \varphi$ for any $\varphi$. We have

$$
\begin{aligned}
\left\|P_{j} R(t) \varphi\right\|^{2} & =\left\|V(t) \int_{0}^{t} V^{-1}(s) P_{j} W^{o}(s) U(s) \varphi d s\right\|^{2} \\
& \leqslant\left(\int_{0}^{t}\left\|P_{j} W^{o}(s)\right\| d s\right)^{2}\|\varphi\|^{2}
\end{aligned}
$$

If $\mathrm{H} 3$ (i) holds, using (2.26) with $q=1 / 2$, we get

$$
\begin{aligned}
\sum_{j=1}^{\infty} \lambda_{j}\left\|P_{j} R(t) \varphi\right\|^{2} & \leqslant \sum_{j=1}^{\infty}\left(\int_{0}^{l} \lambda_{j}^{1 / 2}\left\|P_{j} W^{o}(s)\right\| d s\right)^{2}\|\varphi\|^{2} \\
& \leqslant\left(\int_{0}^{t}\left\|W^{o}(s)\right\|_{2, H^{1 / 2}} d s\right)^{2}\|\varphi\|^{2}<\infty
\end{aligned}
$$

This means that for any $t \geqslant 0$,

$$
R(t): \quad \mathscr{H} \rightarrow D\left(H^{1 / 2}\right)
$$

hence

$$
U(t): \quad D\left(H^{1 / 2}\right) \rightarrow D\left(H^{1 / 2}\right)
$$


The positivity of $H$ implies that for any $\varphi, \chi \in D\left(H^{1 / 2}\right)$ (see ref. 14, p. 53)

$$
2|\langle\chi \mid H \varphi\rangle| \leqslant\langle\chi \mid H \chi\rangle+\langle\varphi \mid H \varphi\rangle
$$

so that using (2.13), we can write for any $\varphi \in D\left(H^{1 / 2}\right),\|\varphi\|=1$,

$$
\begin{aligned}
\langle H\rangle_{\varphi}(t)= & \langle U(t) \varphi \mid H U(t) \varphi\rangle \\
= & \langle V(t) \varphi \mid H V(t) \varphi\rangle \\
& +2 \operatorname{Re}\langle V(t) \varphi \mid H R(t) \varphi\rangle+\langle R(t) \varphi \mid H R(t) \varphi\rangle \\
\leqslant & 2(\langle V(t) \varphi \mid H V(t) \varphi\rangle+\langle R(t) \varphi \mid H R(t) \varphi\rangle) \\
= & 2\left(\left\|H^{1 / 2} V(t) \varphi\right\|^{2}+\left\|H^{1 / 2} R(t) \varphi\right\|^{2}\right)
\end{aligned}
$$

The estimate (2.29) together with (2.33) yields for the first term of (2.39)

$$
\begin{aligned}
\left\|H^{1 / 2} V(t) \varphi\right\|^{2} & =\sum_{j=1}^{\infty}\left\|H_{j}^{1 / 2} V(t) \varphi\right\|^{2} \\
& \leqslant s \sum_{j=1}^{\infty} \lambda_{j}\left\|P_{j} V(t) \varphi\right\|^{2} \leqslant s\left\langle H^{1 / 2} \varphi \mid H^{1 / 2} \varphi\right\rangle
\end{aligned}
$$

which is independent of time. We deal with the second term as follows. For any $N \in \mathbf{N}^{*}$, we have by virtue of (2.34) and (2.14)

$$
\begin{aligned}
& \left\|H^{1 / 2} R(t) \varphi\right\|^{2} \\
& \quad=\sum_{j=1}^{N}\left\|H_{j}^{1 / 2} R(t) \varphi\right\|^{2}+\sum_{j=N+1}^{\infty}\left\|H_{j}^{1 / 2} R(t) \varphi\right\|^{2} \\
& \quad \leqslant s \lambda_{N} \sum_{j=1}^{N}\left\|P_{j} R(t) \varphi\right\|^{2}+s \sum_{j=N+1}^{\infty} \lambda_{j}\left\|P_{j} R(t) \varphi\right\|^{2} \\
& \quad \leqslant 4 s \lambda_{N}+s \sum_{j=N+1}^{\infty} \lambda_{j}\left[\int_{0}^{t}\left\|P_{j} W^{o}(s)\right\| d s\right]^{2}
\end{aligned}
$$

Gathering these estimates, we finally get for any $\varphi \in D\left(H^{1 / 2}\right),\|\varphi\|=1$,

$$
\langle H\rangle_{\varphi}(t) \leqslant 2 s\left\{\left\|H^{1 / 2} \varphi\right\|^{2}+4 \lambda_{N}+\sum_{j=N+1}^{\infty} \lambda_{j}\left[\int_{0}^{l}\left\|P_{j} W^{o}(s)\right\| d s\right]^{2}\right\}
$$


Assume H3(i) holds.

Applying (2.26) to the last term of (2.42), we get, using the monotonicity of $\lambda_{j}$ in $j \in \mathbf{N}^{*}$,

$$
\begin{aligned}
& \sum_{j=N+1}^{\infty}\left(\int_{0}^{t} \lambda_{j}^{1 / 2}\left\|P_{j} W^{o}(s)\right\| d s\right)^{2} \\
& \leqslant \frac{1}{\lambda_{N+1}^{2 q-1}} \sum_{j=N+1}^{\infty}\left(\int_{0}^{l} \lambda_{j}^{q}\left\|P_{j} W^{o}(s)\right\| d s\right)^{2} \\
& \leqslant \frac{1}{\lambda_{N+1}^{2 q-1}}\left\{\int_{0}^{l}\left[\sum_{j=N+1}^{\infty} \lambda_{j}^{2 q}\left\|P_{j} W^{o}(s)\right\|^{2}\right]^{1 / 2} d s\right\}^{2} \\
& \leqslant \frac{\left(\int_{0}^{t}\left\|W^{o}(s)\right\|_{2, H^{4}} d s\right)^{2}}{\lambda_{N+1}^{2 q-1}}
\end{aligned}
$$

Thus, for some constant $c<\infty$ independent of $t$ and $N$, we have the estimate

$$
\langle H\rangle_{\varphi}(t) \leqslant c\left(1+\lambda_{N}+\frac{\left(\int_{0}^{t}\left\|W^{o}(s)\right\|_{2, H^{q}} d s\right)^{2}}{\lambda_{N+1}^{2 q-1}}\right)
$$

If

$$
\lim _{N \rightarrow \infty} \sup _{s \in \mathbf{R}^{+}}\left|\sum_{j=1}^{N} \lambda_{j}^{2 q}\left\|P_{j} W^{o}(s)\right\|^{2}-\left\|W^{o}(s)\right\|_{2, H^{q}}^{2}\right|=0
$$

we have

$$
\begin{aligned}
\frac{1}{t} \int_{0}^{t} & {\left[\sum_{j=N+1}^{\infty} \lambda_{j}^{2 q}\left\|P_{j} W^{o}(s)\right\|^{2}\right]^{1 / 2} d s } \\
& \leqslant \frac{1}{t} \int_{0}^{t}\left[\sup _{s \in \mathbf{R}^{+}}\left|\sum_{j=1}^{N} \lambda_{j}^{2 q}\left\|P_{j} W^{o}(s)\right\|^{2}-\left\|W^{o}(s)\right\|_{2 . H^{4}}^{2}\right|\right]^{1 / 2} d s \\
& =\left[\sup _{s \in \mathbf{R}^{+}} \mid \sum_{j=1}^{N} \lambda_{j}^{2 q}\left\|P_{j} W^{o}(s)\right\|^{2}-\left\|W^{o}(s)\right\|_{2 . H^{4}}^{2}\right]^{1 / 2} \equiv \sqrt{g(N+1)}
\end{aligned}
$$

where $g(N) \rightarrow 0$ as $N \rightarrow \infty$ and $g(N)>0$ for any $N$, due to the last condition in $\mathrm{H} 2$. Thus

$$
\langle H\rangle_{\varphi}(t) \leqslant c\left(1+\lambda_{N}+\frac{t^{2}}{\lambda_{N+1}^{2 g-1}} g(N+1)\right)
$$


Assume H3(ii) holds.

In this case,

$$
\begin{aligned}
& \sum_{j=N+1}^{\infty} \lambda_{j}\left[\int_{0}^{t}\left\|P_{j} W^{o}(s)\right\| d s\right]^{2} \\
& \leqslant \sum_{j=N+1}^{\infty}\left[\int_{0}^{t}|k(s)| d s\right]^{2} \lambda_{j}\left\|P_{j} \omega^{o}\right\|^{2} \\
& \leqslant \frac{\left[\int_{0}^{t}|k(s)| d s\right]^{2}}{\lambda_{N+1}^{2 g-1}} \sum_{j=N+1}^{\infty} \lambda_{j}^{2 q}\left\|P_{j} \omega^{o}\right\|^{2}
\end{aligned}
$$

Hence,

$$
\langle H\rangle_{\varphi}(t) \leqslant c\left(1+\lambda_{N}+\frac{\left[\int_{0}^{\prime}|k(s)| d s\right]^{2}}{\lambda_{N+1}^{2 q-1}} g(N+1)\right)
$$

where

$$
0<g(N)=\sum_{j=N}^{\infty} \lambda_{j}^{2 q}\left\|P_{j} \omega^{o}\right\|^{2} \geqslant g(N+1) \rightarrow 0 \quad \text { as } \quad N \rightarrow \infty
$$

It remains to minimize the right-hand sides of (2.44), (2.47), and (2.49) with respect to $N$ as $t \rightarrow \infty$.

Lemma 2.3. Let $\alpha \geqslant 0$ and $r(t)$ be a positive function of $t \in \mathbf{R}^{+}$such that $\lim _{t \rightarrow \infty} r(t)=\infty$. Let $\lambda_{j}$ and $g(j)$ be two sequences of positive numbers such that $\lambda_{j}<\lambda_{j+1} \rightarrow \infty$ as $j \rightarrow \infty$ and $g(j) \geqslant g(j+1)$. Consider

$$
h(N, t) \equiv \lambda_{N}+\frac{r(t)}{\lambda_{N+1}^{\alpha}} g(N+1)
$$

Then, for each $t$, there exists a unique $N(t) \in \mathbf{N}^{*}$ such that in the limit $t \rightarrow \infty$

$$
h(N(t), t)=o\left(r^{1 /(1+\alpha)}(t)\right)
$$

if $\lim _{j \rightarrow \infty} g(j)=0$ and

$$
h(N(t), t)=O\left(r^{1 /(1+\alpha)}(t)\right)
$$

if $\inf _{j \in N^{*}} g(j)>0$. 
Remarks. If $\sup _{t \in \mathbf{R}^{+}} r(t)<\infty$, we get

$$
\sup _{t \in \mathbf{R}^{+}} h\left(N_{0}, t\right)<\infty
$$

for any fixed $N_{0} \in \mathbf{N}^{*}$.

This lemma is optimal in the sense that any asymptotically different choice of $N(t)$ as $t \rightarrow \infty$ yields a weaker estimate, as easily verified from the proof.

Proof. The hypotheses on $\lambda_{j}$ and $g(j)$ imply that

$$
\lambda_{j} / g^{1 /(1+\alpha)}(j)<\lambda_{j+1} / g^{1 /(1+\alpha)}(j+1) \rightarrow \infty \quad \text { as } j \rightarrow \infty
$$

so that there exists a unique index $N(t)$ for any $t \in \mathbf{R}^{+}$such that

$$
\lambda_{N(t)} / g^{1 /(1+\alpha)}(N(t)) \leqslant r^{1 /(1+\alpha)}(t)<\lambda_{N(t)+1} / g^{1 /(1+\alpha)}(N(t)+1)
$$

Hence, taking $N=N(t)$ in $h(N, t)$, we can write

$$
h(N(t), t) \leqslant r^{1 /(1+\alpha)}(t)\left(g^{1 /(1+\alpha)}(N(t))+g^{1 /(1+\alpha)}(N(t)+1)\right)
$$

where $\lim _{t \rightarrow \infty} N(t)=\infty$. Hence, depending on the behavior at infinity of $g(N)$, we get a little- $o$ or a big- $O$ estimate for $h(N(t), t)$.

The theorem is proven by applying this lemma with $\alpha=2 q-1$ and the corresponding $r(t)$ to $(2.44)$, taking $g(N) \equiv 1$, and to $(2.47),(2.49)$ with the $g(N)$ defined in $(2.46),(2.50)$.

\section{UPPER BOUNDS ON $\langle f(H)\rangle_{\varphi}(t)$}

Looking back at Theorem 2.1, we see that the $\lambda_{j}$ play a significant role in the hypotheses on the size of $W^{o}(t)$ only and that the end result on $\langle H\rangle_{\varphi}(t)$ is independent of $\lambda_{j}$. This calls for a generalization to expectation values of operators of the following type.

Hypothesis H4. Let $f: \mathbf{R} \rightarrow \mathbf{R}$ be a function such that

$$
0<f_{j}=\inf _{\lambda \in \sigma_{j}} f(\lambda) \quad \text { and } \quad F_{j}=\sup _{\lambda \in \sigma_{j}} f(\lambda), \quad j \in \mathbf{N}^{*}
$$

satisfy

$$
F_{j}<f_{j+1}, \quad \forall j \in \mathbf{N}^{*} \quad \text { and } \quad \sup _{j \in \mathbf{N}^{*}} F_{j} / f_{j}=c<\infty
$$


In this case, $f(H)$ is positive and self-adjoint on its domain $D(f(H))$, which is dense (ref. $14, \S$ VI.5) and easily shown to coincide with

$$
D(f(H))=\left\{\psi \in \mathscr{H} \mid \sum_{j=1}^{\infty} f_{j}^{2}\left\|P_{j} \psi\right\|^{2}<\infty\right\}
$$

The values of $f$ outside the support of the spectrum of $H$ are irrelevant.

We introduce another set of hypotheses, each of which gives rise to a different estimate.

Hypothesis H5. (i) There exists $q \geqslant 1 / 2$ such that

$$
\left(\sum_{j=1}^{\infty} f_{j}^{2 q}\left\|P_{j} W^{o}(s)\right\|^{2}\right)^{1 / 2} \equiv\left\|W^{o}(t)\right\|_{2 . J^{4}(H)}
$$

is locally integrable as a function of $t$.

(ii) Hypothesis $\mathrm{H} 5(\mathrm{i})$ holds and $W^{o}(t)$ has the form

$$
W^{o}(t)=k(t) \omega^{o}
$$

where $k$ is a real-valued function and $\omega^{\circ}$ is a bounded, self adjoint, timeindependent operator on $\mathscr{H}$.

Then, by repeating the proof of Theorem 2.1, with $f(H)$ in place of $H$, $f_{j}$ in place of $\lambda_{j}$, and $c$ in place of $s$, we get immediately the following result:

Theorem 3.1. Assume hypotheses $\mathrm{H} 1$ and $\mathrm{H} 2$, and let $f: \mathbf{R} \rightarrow \mathbf{R}$ be such that $\mathrm{H} 4$ and $\mathrm{H} 5$ are satisfied. Then, the evolution operator solution of $(2.8)$ is such that for any $t \in \mathbf{R}^{+}$

$$
U(t): \quad D\left(f^{1 / 2}(H)\right) \rightarrow D\left(f^{1 / 2}(H)\right)
$$

Let $\varphi \in D\left(f^{1 / 2}(H)\right)$ and consider $\langle f(H)\rangle_{\varphi}(t)=\langle U(t) \varphi \mid f(H) U(t) \varphi\rangle$ as $t \rightarrow \infty$. If $\mathrm{H} 5$ (i) holds, then

$$
\langle f(H)\rangle_{\varphi}(t)=O\left(\left[\int_{0}^{l}\left\|W^{o}(s)\right\|_{2, f^{q}(H)} d s\right]^{1 / q}\right)
$$

If, in addition, $\sum_{j=1}^{\infty} f_{j}^{2 q}\left\|P_{j} W^{o}(s)\right\|^{2}$ converges uniformly in $s \in \mathbf{R}^{+}$, we get

$$
\langle f(H)\rangle_{\varphi}(t)=o\left(t^{1 / q}\right)
$$


If H5(ii) holds, then

$$
\langle f(H)\rangle_{\varphi}(t)=o\left(\left[\int_{0}^{t}|k(s)| d s\right]^{1 / q}\right)
$$

Using this result, if $\langle H\rangle_{\varphi}(t)$ cannot be estimated by Theorem 2.1, we can nevertheless try to get a bound on the expectation value of $f(H)$, taking an $f$ such that $0<f(H)<H$. We can actually consider the problem from the reversed point of view. Given a perturbation $W(t)$, can we find an unbounded operator whose expectation value can be controlled as $t \rightarrow \infty$ ? This is the object of the following result.

Corollary 3.1. Assume that there exists a positive, strictly increasing sequence $\left\{\mu_{j}\right\}_{j=1}^{\infty}$ such that

$$
\left(\sum_{j=1}^{\infty} \mu_{j}^{2}\left\|P_{j} W(t)\right\|^{2}\right)^{1 / 2}
$$

is locally integrable as a function of $t$.

Then, for any $q \geqslant 1 / 2$ there exists a function $f: \mathbf{R}^{+} \rightarrow \mathbf{R}^{+}$, depending on $q$ and satisfying $\mathrm{H} 4$, such that

$$
\langle f(H)\rangle_{\varphi}(t)=O\left(\left[\int_{0}^{l}\left\|W^{o}(s)\right\|_{2 . f^{\left.\varphi^{(} H\right)}} d s\right]^{1 / q}\right)
$$

for any initial condition in $D\left(f^{1 / 2}(H)\right)$.

Proof. Take the function defined for any $j \in \mathbf{N}^{*}$ by

$$
f(\lambda)= \begin{cases}\mu_{j}^{1 / q} & \text { if } \lambda \in \sigma_{j} \\ 0 & \text { otherwise }\end{cases}
$$

which satisfies $\mathrm{H} 4$, and apply Theorem 3.1.

Remark. In case $W^{o}(t)=k(t) \omega^{\circ}$, we get

$$
\langle f(H)\rangle_{\varphi}(t)=o\left(\left[\int_{0}^{t}|k(s)| d s\right]^{1 / q}\right)
$$

for the same $f$.

The interest of this corollary stems from the fact that for a given $W(t)$, $\langle H\rangle_{\varphi}(t)$ may fail to be bounded uniformly in time, whereas the expectation value of some function of $H$ may be (see the example in the next section). 
Another improvement of these results consists in trying to get estimates on $\langle H\rangle_{\varphi}(t)$, respectively $\langle f(H)\rangle_{\varphi}(t)$, of a more general type than algebraic in $t$. This can be achieved by replacing $\lambda_{j}^{q}$, respectively $f_{j}^{q}$, by $a\left(\lambda_{j}\right)$, respectively $a\left(f_{j}\right)$, in the hypotheses on the size of $W^{o}(t)$, where $a: \mathbf{R}^{+} \rightarrow \mathbf{R}^{+}$is some increasing function. More precisely:

Hypothesis H6. Let

$$
\begin{aligned}
a: & \mathbf{R}^{+} \rightarrow \mathbf{R}^{+} \\
& x \mapsto a(x)
\end{aligned}
$$

be a continuous, strictly monotonic increasing function such that $b$ defined by

$$
\begin{aligned}
b: & {\left[\beta, \infty\left[\rightarrow \mathbf{R}^{+}\right.\right.} \\
& x \mapsto a^{2}(x) / x
\end{aligned}
$$

is nondecreasing, for some $\beta>0$.

We indicate in an appendix how to alter the proof of Theorem 2.1 in order to show the following result:

Theorem 3.2. Assume $\mathrm{H} 1$ and $\mathrm{H} 2$ and let $f$ and $a$ be two functions satisfying $\mathrm{H} 4$ and $\mathrm{H} 6$. If

$$
\text { (i) }\left(\sum_{j=1}^{\infty} a^{2}\left(f_{j}\right)\left\|P_{j} W(t)\right\|^{2}\right)^{1 / 2} \equiv\left\|W^{o}(t)\right\|_{2, a \cdot f(H)}
$$

is locally integrable as a function of $t$, respectively

$$
\text { (ii) } W^{o}(t)=k(t) \omega^{o}
$$

where $k(t)$ is a real-valued function and $\omega^{\circ}$ is a bounded, self-adjoint, timeindependent operator and $W^{\prime \prime}(t)$ satisfies (i), then, as $t \rightarrow \infty$,

$$
\text { (i) }\langle f(H)\rangle_{\varphi}(t)=O\left(a^{-1}\left[\int_{0}^{t}\left\|W^{o}(s)\right\|_{2, a \circ f(H)} d s\right]\right)
$$

respectively

$$
\text { (ii) }\langle f(H)\rangle_{\varphi}(t)=O\left(a^{-1}\left[\int_{0}^{t}|k(s)| d s\right]\right)
$$

for any initial condition $\varphi \in D\left(f^{1 / 2}(H)\right)$. 
This last result shows more precisely how the strength of the hypotheses, characterized by the function $a \circ f$, can be modulated in order to improve the time dependence of the estimates, characterized by $a$, or to enlarge the class of operators to which the results apply, characterized by $f$.

\section{EXAMPLE}

Let us demonstrate the use of the estimates of the preceding section on a specific example.

Assume $m_{j}=1 \forall j \in \mathbf{N}^{*}$ and consider

$$
H=\sum_{j=1}^{\infty} \lambda_{j}\left|\varphi_{j}\right\rangle\left\langle\varphi_{j}\right| \equiv \sum_{j=1}^{\infty} \lambda_{j} P_{j} \quad \text { where } \quad \lambda_{j}=j^{\alpha}, \quad \alpha>0
$$

We define

$$
\chi(t)=\sum_{j=1}^{\infty} c_{j}(t) \varphi_{j} \quad \text { with } \quad c_{j}(t)=\frac{1}{j^{\beta-f(t)}}, \quad \beta>0
$$

and

$$
f(t)=\beta-\alpha / 2-1 / 2-1 /\left[2(1+t)^{2 n}\right], \quad n>0
$$

Then $\sup _{t \in \mathbf{R}^{+}}\|\chi(t)\|<\infty$ (see below) and actually it is not difficult to see that $\chi(t)$ is analytic in a sector containing $\mathbf{R}^{+}$. Let $g(t)$ be a $C^{\infty}$ real-valued function such that

$$
\sup _{t \in \mathbf{R}^{+}}|g(t)|=\infty, \quad g(t) \in L^{1}\left(\mathbf{R}^{+}\right), \quad|g(t)|(1+t)^{n} \notin L^{1}\left(\mathbf{R}^{+}\right)
$$

This is the case with $n=1$ if

$$
\begin{gathered}
\operatorname{supp}|g(t)| \subseteq\left\{t|| t-j \mid \leqslant 1 / j^{3}, \forall j \in \mathbf{N}^{*}\right\} \\
\sup _{t \in\left\{t|| t-j \mid \leqslant 1 / j^{3}\right\}}|g(t)|=j=|g(s)| \quad \forall s \in\left\{s|| s-j \mid \leqslant 1 /\left(2 j^{3}\right)\right\},
\end{gathered}
$$

$$
\forall j \in \mathbf{N}^{*}
$$

Then we set

$$
W(t)=g(t)|\chi(t)\rangle\langle\chi(t)|
$$

By construction, $W(t)$ satisfies $\mathrm{H} 2$ and the trivial bound (1.9) cannot be applied for this bounded perturbation. With the definition

$$
W^{o}(t)=g(t)\left(|\chi(t)\rangle\left\langle\chi(t)\left|-\sum_{j=1}^{\infty} P_{j}\right| \chi(t)\right\rangle\langle\chi(t)| P_{j}\right)
$$


we easily get

$$
\left\|P_{j} W^{o}(t)\right\|=g(t) c_{j}(t)\left(\sum_{\substack{k=1 \\ k \neq j}}^{\infty} c_{k}^{2}(t)\right)^{1 / 2}
$$

We show the following result below.

Lemma 4.1. There exist $t$-independent constants $0<p_{-}<p_{+}$such that

$$
p_{-} g(t) c_{j}(t) \leqslant\left\|P_{j} W^{o}(t)\right\| \leqslant p_{+} g(t) c_{j}(t)
$$

Hence, using the integral test, we obtain that the behavior of $\left\|W^{o}(t)\right\|_{2, H^{1 / 2}}$ is equivalent to that of

$$
\begin{aligned}
|g(t)|\left(\sum_{j=1}^{\infty} \lambda_{j} c_{j}^{2}(t)\right)^{1 / 2} & =|g(t)|\left(\sum_{j=1}^{\infty} \frac{1}{j^{2(\beta-f(t))-\alpha}}\right)^{1 / 2} \\
& \geqslant|g(t)|\left(\frac{1}{2(\beta-f(t))-\alpha-1}\right)^{1 / 2}=|g(t)|(1+t)^{n}
\end{aligned}
$$

By assumption, $|g(t)|(1+t)^{n}$ fails to be integrable on $\mathbf{R}^{+}$, so that we cannot show that $\sup _{\boldsymbol{t} \in \mathbf{R}^{+}}\langle H\rangle_{\varphi}(t)<\infty$. However, considering $f(H) \equiv H^{\gamma}$ with $0<\gamma<1$, we find that the behavior of $\left\|W^{o}(t)\right\|_{1, H^{\prime / 2}}$ is equivalent to that of

$$
\begin{aligned}
|g(t)| & \left(\sum_{j=1}^{\infty} \lambda_{j}^{\gamma} c_{j}^{2}(t)\right)^{1 / 2} \\
& =|g(t)|\left(\sum_{j=1}^{\infty} \frac{1}{j^{2(\beta-f(t))-\alpha \gamma}}\right)^{1 / 2} \\
& \leqslant|g(t)|\left(1+\frac{1}{2(\beta-f(t))-\gamma \alpha-1}\right)^{1 / 2} \\
& =|g(t)|\left(1+\frac{1}{(1-\gamma) \alpha+1 /(1+t)^{2 n}}\right)^{1 / 2}
\end{aligned}
$$

which is integrable on $\mathbf{R}^{+}$. Consequently, $\left.\forall \gamma \in\right] 0,1\left[\right.$ and $\forall \varphi \in D\left(H^{\gamma / 2}\right)$,

$$
\sup _{t \in \mathbf{R}^{+}}\left\langle H^{\prime}\right\rangle_{\varphi}(t)<\infty
$$


Proof of Lemma 4.1. On the one hand, using $2(\beta-f(t))>1+\alpha$, we find

$$
\sum_{\substack{k=1 \\ k \neq j}}^{\infty} c_{k}^{2}(t) \leqslant \sum_{k=1}^{\infty} c_{k}^{2}(t) \leqslant \sum_{k=1}^{\infty} 1 / j^{1+\alpha}<\infty
$$

On the other hand, using the integral test, we obtain

$$
\begin{aligned}
\sum_{\substack{k=1 \\
k \neq j}}^{\infty} c_{k}^{2}(t) & =\sum_{k=1}^{j-1}+\sum_{k=j+1}^{\infty} c_{k}^{2}(t) \\
& \geqslant \int_{1}^{j}+\int_{j+1}^{\infty} \frac{d x}{x^{2(\beta-f(t))}} \\
& =\frac{1}{2(\beta-f(t))-1}\left(1+\frac{1}{(j+1)^{2(\beta-f(t))-1}}-\frac{1}{j^{2(\beta-f(t))-1}}\right)(4 .)
\end{aligned}
$$

If $j=1$, since $2[\beta-f(t)]-1<1+\alpha$, we can take $1 /\left[(1+\alpha) 2^{1+x}\right]$ as a lower bound on (4.14). If $j>1$, then

$$
1-\frac{1}{j^{2(\beta-f(t))-1}}>1-\frac{1}{2^{\alpha}}
$$

yields $\left.\left(2^{\alpha}-1\right) /[1+\alpha) 2^{\alpha}\right]>0$ as a lower bound.

\section{APPLICATIONS}

In this section, we consider a restricted class of Hamiltonians $H+W(t)$ which are likely to appear in specific models and fit in the framework described above. For this class of Hamiltonians it is even possible to improve slightly the results of Theorem 2.1 .

Proposition 5.1. Assume $\mathrm{H} 1$ and $\mathrm{H} 2$ and let $H$ be such that

$$
\lambda_{j} \leqslant l^{x} \quad \text { as } \quad j \rightarrow \infty
$$

for $l, \alpha>0$ independent of $j$, and let $W^{\circ}(t)$ be such that

$$
\sup _{t \in \mathbf{R}^{+}}\left\|P_{j} W^{o}(t)\right\| \leqslant c / j^{\beta}
$$

for $c, \beta>0$ independent of $j$. Then, provided $2 \beta-\alpha>1$, we get for any $\varphi \in D\left(H^{1 / 2}\right)$,

$$
\langle H\rangle_{\varphi}(t)=O\left(t^{\alpha /(\beta-1 / 2)}\right)
$$


Remarks. 1. Applying the results of Section 2, we get under the same conditions $\langle H\rangle_{\varphi}(t)=o\left(t^{\delta}\right)$ with $\delta=\alpha /(\beta-1 / 2)+\varepsilon$, for arbitrary small $\varepsilon>0$.

2. The exponent $\alpha$ is characteristic of $H$, whereas $\beta$ characterizes $W(t)$. If $\alpha$ gets smaller, the exponent in (5.3) gets better, independently of $W(t)$. However, taking higher values for $\alpha$ requires taking weaker perturbations.

3. Replacing $H$ by some power of $H$ merely amounts here to altering the exponent $\alpha$ appropriately.

Proof. In order to show the proposition, we simply estimate more carefully the last term in Eq. (2.42) using our hypotheses. We can write

$$
\begin{aligned}
\sum_{j=N+1}^{\infty} \lambda_{j}\left(\int_{0}^{t}\left\|P_{j} W^{o}(s)\right\| d s\right)^{2} & \leqslant l c t^{2} \sum_{j=N+1}^{\infty} 1 / j^{2 \beta-\alpha} \\
& \leqslant \bar{c} t^{2} /(N+1)^{2 \beta-\alpha-1}
\end{aligned}
$$

for some constant $\bar{c}$. Hence we are led to minimize

$$
N^{\alpha}+t^{2} /(N+1)^{2 \beta-\alpha-1}
$$

by means of Lemma 2.3, which yields the result.

Let us consider a class of perturbations $W(t)$ for which the estimate (5.2) can be proven. This class of operators was introduced recently in ref. 10 .

Assume $m_{j}<\infty \forall j \in \mathbf{N}^{*}$ and denote by

$$
\left\{\varphi_{j}^{r}\right\}_{j \in \mathbb{N}^{*}}^{r=1, \ldots m_{j}}
$$

an orthonormal basis of eigenvectors of $H$ such that $P_{j} \varphi_{j}^{r}=\varphi_{j}^{r}, r=1, \ldots, m_{j}$. An operator $A$ belongs to the class $\chi(p, \gamma)$, where $p, \gamma \in \mathbf{R}$, if there exists a constant $C$ such that

$$
\left|\left\langle\varphi_{j}^{r} \mid A \varphi_{k}^{s}\right\rangle\right| \leqslant \frac{C}{\langle k-j\rangle^{p}(k j)^{\gamma}}
$$

for all $r=1, \ldots, m_{j}, s=1, \ldots, m_{k}$, where $\langle n\rangle=|n|$ if $n \neq 0$, and $\langle 0\rangle=1$.

Table I. Bounds on $\left\|P_{j} W^{o}(t)\right\|$ for $\delta=0$

\begin{tabular}{cccccc}
\hline & $p<0$ & $p=0$ & $0<p<1$ & $p=1$ & $p>1$ \\
\hline $0<\gamma<1$ & - & - & $1 / j^{2 \gamma+p-1}$ & $\ln j / j^{2 \gamma}$ & $1 / j^{2 \gamma}$ \\
$\gamma=1$ & - & - & $\ln j / j^{1+p}$ & $\ln j / j^{2}$ & $1 / j^{2}$ \\
$\gamma>1$ & $1 / j^{\gamma+p}$ & $1 / j^{\gamma}$ & $1 / j^{\gamma+p}$ & $1 / j^{\gamma+1}$ & $1 / j^{\gamma+\min (p, \gamma)}$ \\
\hline
\end{tabular}


Table II. Bounds on $\left\|P_{j} W^{\circ}(t)\right\|$ for $\delta>0$

\begin{tabular}{|c|c|c|c|c|c|}
\hline & $p<0$ & $p=0$ & $0<p<1$ & $p=1$ & $p>1$ \\
\hline$\gamma^{\prime} \leqslant 0$ & - & - & - & - & $1 / j^{y^{\prime}+\min \left(p . r^{\prime}\right)}$ \\
\hline $0<\gamma^{\prime}<1$ & - & - & $\begin{array}{c}1 / j^{\gamma^{\prime}+p} \text { if } p<\gamma \text { and } \gamma \geqslant 1 \\
1 / j^{\gamma^{\prime}+\gamma+p-1} \text { if } p \geqslant \gamma \text { or } p<\gamma<1\end{array}$ & $\begin{array}{c}1 / j^{\gamma^{\prime}+1} \text { if } 1<\gamma \\
\ln j / j^{\gamma+\gamma} \text { if } l \geqslant \gamma\end{array}$ & $1 / j^{y^{\prime}+\min (p, \ddot{\prime})}$ \\
\hline$\gamma^{\prime}=1$ & - & - & $1 / j^{1+p}$ & $1 / j^{2}$ & $1 / j^{1+\min (\rho . y)}$ \\
\hline$\gamma^{\prime}>1$ & $1 / j^{\prime+p}$ & $1 / j^{\prime}$ & $1 / j^{y^{\prime}+p}$ & $1 / j^{y^{\prime}+1}$ & $\begin{array}{l}1 / j^{r^{\prime}+P} \text { if } p<\gamma^{\prime} \\
1 / j^{r^{\prime}+\gamma} \text { if } p \geqslant \gamma^{\prime}\end{array}$ \\
\hline
\end{tabular}

We prove the following technical lemma in an appendix, using the Schur condition.

Lemma 5.1. Assume $m_{j} \leqslant M j^{\delta}$, where $M$ and $\delta \geqslant 0$ are independent of $j$, and let $W^{o}(t)$ belong to the class $\chi(p, \gamma)$, with a constant $C$ uniform in $t \in \mathbf{R}^{+}$, where $\gamma>0$ and $\gamma-\delta+p>1$. Then, there exists a constant $\kappa$ such that:

(i) If $\delta=0,\left\|P_{j} W^{o}(t)\right\|$ is bounded by $\kappa^{-}$times the quantities in Table I.

(ii) If $\delta>0$, with $\gamma^{\prime}=\gamma-\delta,\left\|P_{j} W^{o}(t)\right\|$ is bounded by $\kappa$ times the quantities in Table II.

Remarks. 1. We can get rid of the logarithms in Tables I and II by decreasing the exponent of $j$ by an arbitrarily small amount.

2. In order to apply Proposition 5.1, we further need the exponent of $j$ to be greater than $1 / 2$.

\section{CONCLUDING REMARKS}

Here we make explicit some links between our results on expectation values of $H$ or $f(H)$ and other relevant quantities in the study of quantum stability. Let us recall these notions and their properties, following mainly refs. 5,1 , and 19. Consider the subspaces

$$
\begin{gathered}
\mathscr{H}^{p}(U)=\{\psi \in \mathscr{H} \mid\{U(t) \psi \mid t \geqslant 0\} \text { is precompact in } \mathscr{H}\} \\
\mathscr{H}^{f}(U)=\left\{\left.\varphi \in \mathscr{H}\right|_{\tau \rightarrow \infty} \frac{1}{\tau} \int_{0}^{\tau}\|K U(t) \varphi\|^{2} d t=0\right. \\
\text { for any compact operator } K\}
\end{gathered}
$$


Regardless of the time dependence of the Hamiltonian, $\mathscr{H}^{p}(U)$ and $\mathscr{H}^{f}(U)$ are closed subspaces of $\mathscr{H}$ and $\mathscr{H}^{p}(U) \perp \mathscr{H}^{f}(U){ }^{(5,19)}$ D'Oliveira further introduced subspaces related to some operator. Let $A$ defined on $D(A)$ be unbounded, self-adjoint, and positive with discrete spectrum and assume that $U(t): D(A) \rightarrow D(A), \forall t \in \mathbf{R}^{+}$. We set

$$
\begin{aligned}
& \mathscr{S}^{b d}(A)=\left\{\varphi \in D(A) \mid \sup _{t \in \mathbf{R}^{*}}\langle A\rangle_{\varphi}(t)<\infty\right\} \\
& \mathscr{S}^{\text {unn }}(A)=D(A) \cap\left[\mathscr{S}^{b d}(A)\right]^{\perp}
\end{aligned}
$$

It is shown in ref. 19 that

$$
\mathscr{S}^{b d l}(A) \subset \mathscr{H}^{p}(U) \quad \text { and } \quad D(A) \cap \mathscr{H}^{f}(U) \subset \mathscr{S}^{u n}(A)
$$

If we restrict our concern to time-periodic Hamiltonians, i.e., $W(t+1)=W(t)$, and assume that

$$
m_{j}<\infty, \quad \forall j \in \mathbf{N}^{*}
$$

$\operatorname{then}^{(5.1)}$

$$
\mathscr{H}=\mathscr{H}^{p}(U) \oplus \mathscr{H}^{f}(U)
$$

with

$$
\mathscr{H}^{\prime}(U)=\mathscr{H}_{c}(U(1)) \quad \text { and } \quad \mathscr{H}^{p}(U)=\mathscr{H}_{p p}(U(1))
$$

where $\mathscr{H}_{c}(U(1))$, respectively $\mathscr{H}_{p p}(U(1))$, are the continuous, respectively pure point, spectral subspaces of the monodromy operator $U(1)$. Moreover, as implicitly contained in refs. 5 and 1, we deduce from (6.5) with $A=H$ that

$$
\varphi \in D(H) \cap \mathscr{H}_{\boldsymbol{c}}(U(1)) \Rightarrow \sup _{t \in \mathbf{R}^{*}}\langle H\rangle_{\varphi}(t)=\infty
$$

or, equivalently,

$$
\varphi \in D(H) \quad \text { and } \quad \sup _{t \in \mathbf{R}^{*}}\langle H\rangle_{\varphi}(t)<\infty \Rightarrow \varphi \in \mathscr{H}_{p p}(U(\mathbf{1}))
$$

Remark that $U(t): D(H) \rightarrow D(H), \forall t \in \mathbf{R}^{+}$, under our general hypotheses. The converse statement to $(6.10)$ is believed to be false, although no proof seems to be available yet. ${ }^{(1.13)}$ We can replace $H$ by $f(H)$ [choosing $A=f(H)]$ in $(6.9),(6.10)$ under the hypotheses of Theorem 3.1 and (6.6), 
provided we take initial conditions $\varphi \in D\left(f^{1 / 2}(H)\right)$ instead of $\varphi \in D(f(H))$. The question of domains is then solved by the property $U(t)$ : $D\left(f^{1 / 2}(H)\right) \rightarrow D\left(f^{1 / 2}(H)\right), \forall t \in \mathbf{R}^{+}$, which alters neither the proof nor the results of ref. 19. Hence, the study of expectation values may give information on the spectral properties of the monodromy operator in such cases. (Note, however, that Theorems 2.1 and 3.1 cannot yield boundedness of expectation values in the time-periodic case.)

In case of arbitrary time dependence, the Hilbert space cannot be decomposed according to (6.7), but we can write

$$
\mathscr{H}=\mathscr{H}^{p}(U) \oplus \mathscr{H}^{f}(U) \oplus \mathscr{H}^{a}(U)
$$

where $\mathscr{H}^{a}(U)$ is called the "U-unusual" subspace in ref. 19. To show the existence of $\mathscr{H}^{a}(U)$, d'Oliveira(19) studies an explicit example of a Hamiltonian for which he proves that actually

$$
\mathscr{H}=\mathscr{H}^{a}(U)
$$

The example treated in Section 4 yields in turn a case where

$$
\mathscr{H}=\mathscr{H}^{P}(U)
$$

Indeed, for any $\varphi$ in the dense domain $D\left(H^{y / 2}\right)$ we showed that

$$
\sup _{t \in \mathbb{R}^{*}}\left\langle H^{\prime}\right\rangle_{\varphi}<\infty
$$

Thus, by virtue of (6.5) and the closedness of $\mathscr{H}^{p}(U)$ we get the result.

Actually, it is not difficult to find a whole class of systems for which

$$
\mathscr{H}=\mathscr{H}^{p}(U)
$$

Consider

$$
H(t)=H+k(t) \omega^{\prime \prime}
$$

where we suppose that $k(t) \in L^{1}$ and that (6.6) holds. Further assume that there exists a positive, strictly increasing sequence $\left\{\mu_{j}\right\}_{j \in \mathrm{N}^{*}}$ such that

$$
\sum_{j=1}^{\infty} \mu_{j}^{2}\left\|P_{j} \omega^{o}\right\|^{2}<\infty
$$

Then, as a consequence of the remark following Corollary 3.1 , we get for any $\varphi$ in the dense domain $D\left(f^{1 / 2}(H)\right)$

$$
\sup _{i \in \mathbf{R}^{*}}\langle f(H)\rangle_{\varphi}<\infty
$$


where $f\left(\sigma_{j}\right)=\left\{\mu_{j}^{2}\right\}, \forall j \in \mathbf{N}^{*}$ and $f\left(\mathbf{R} \backslash \sigma_{j}\right)=\{0\}$. Again, (6.5) and the closedness of $\mathscr{H}^{p}(U)$ yield the result. Hence, the property (6.12) does not seem to be the rule for driven quantum systems; see the conclusion of ref. 19.

\section{APPENDIX A. PROOF OF LEMMA 2.1}

Let us show that $W^{d}(t)$ is $H$-bounded with relative bound $b$. Let $\varphi \in D(H)$; we have

$$
\begin{aligned}
\left\|W^{\prime}(t) \varphi\right\|^{2} & =\sum_{j=1}^{\infty}\left\|P_{j} W(t) P_{j} \varphi\right\|^{2} \\
& \leqslant \sum_{j=1}^{\infty}\left\|W(t) P_{j} \varphi\right\|^{2} \\
& \leqslant \sum_{j=1}^{\infty}\left(b\left\|H P_{j} \varphi\right\|+a(t)\left\|P_{j} \varphi\right\|\right)^{2} \\
& =b^{2}\|H \varphi\|^{2}+a(t)^{2}\|\varphi\|^{2}+2 \sum_{j=1}^{\infty} b\left\|P_{j} H \varphi\right\| a(t)\left\|P_{j} \varphi\right\| \\
& \leqslant(b\|H \varphi\|+a(t)\|\varphi\|)^{2}
\end{aligned}
$$

Consider the strongly $C^{1}$ bounded symmetric operator

$$
W^{N}(t)=\sum_{j=1}^{N} P_{j} W(t) P_{j}
$$

for any $t \in \mathbf{R}^{+}$. We define $K=[0, T]$ such that $t \in K \subset \mathbf{R}^{+}$. We have for $M \geqslant N$ and $\varphi \in D$

$$
\begin{aligned}
& \left\|\left(W^{N}(t)-W^{M}(t)\right) \varphi\right\|^{2} \\
& =\sum_{j=N}^{M}\left\|P_{j} W(t) P_{j} \varphi\right\|^{2} \\
& =\sum_{j=N}^{M}\left\|P_{j} W(t) H^{-1} P_{j} H \varphi\right\|^{2} \\
& \quad \leqslant \sup _{i \in K \subset \mathbf{R}^{+}}\left\|W(t) H^{-1}\right\|^{2} \sum_{j=N}^{M}\left\|P_{j} H \varphi\right\|^{2}
\end{aligned}
$$


Hence $W_{N}(t)$ tends strongly to $W^{d}(t)$, uniformly in $t \in K$, and $W^{d}(t)$ is symmetric. Similarly, for $t \in K \subset \mathbf{R}^{+}$,

$$
\begin{aligned}
\left\|\left(W^{N^{\prime}}(t)-W^{M^{\prime}}(t)\right) \varphi\right\|^{2} & =\sum_{j=N}^{M}\left\|P_{j} W^{\prime}(t) H^{-1} P_{j} H \varphi\right\|^{2} \\
& \leqslant \sup _{t \in K \subset \mathbf{R}^{+}}\left\|W^{\prime}(t) H^{-1}\right\|^{2} \sum_{j=N}^{M}\left\|P_{j} H \varphi\right\|^{2}
\end{aligned}
$$

so that the strongly continuous operator

$$
W^{N^{\prime}}(t)=\sum_{j=1}^{N} P_{j} W^{\prime}(t) P_{j}
$$

tends on $D$ to a limit $w(t)$ uniformly in $t \in K$. Hence $w(t)$ is strongly continuous.

Finally, for any $\varphi \in D$ and $t \in K \subset \mathbf{R}^{+}$,

$$
\left(W^{d}(t)-W^{d}(0)\right) \varphi=\lim _{N \rightarrow \infty} \sum_{j=1}^{N} \int_{0}^{t} P_{j} W^{\prime}(s) H^{-1} P_{j} H \varphi
$$

where

$$
\begin{aligned}
\lim _{N \rightarrow \infty} & \left\|\sum_{j=1}^{N} \int_{0}^{l} P_{j} W^{\prime}(s) P_{j} \varphi-\int_{0}^{\prime} w(s) \varphi d s\right\| \\
& \leqslant \lim _{N \rightarrow \infty} \int_{0}^{\prime}\left(\sum_{j=N+1}^{\infty}\left\|P_{j} W^{\prime}(s) H^{-1} P_{j} H \varphi\right\|^{2}\right)^{1 / 2} d s \\
& \leqslant \int_{0}^{\prime}\left\|W^{\prime}(s) H^{-1}\right\| d s \lim _{N \rightarrow \infty}\left(\sum_{j=N+1}^{\infty}\left\|P_{j} H \varphi\right\|^{2}\right)^{1 / 2}=0
\end{aligned}
$$

This means that

$$
W^{d}(t) \varphi=W^{d}(0) \varphi+\int_{0}^{t} w(s) \varphi d s
$$

so that $W^{\prime l}(t)$ is strongly $C^{1}$.

\section{APPENDIX B. PROOF OF LEMMA 2.2}

We have

$$
\sum_{j=1}^{\infty} \lambda_{j}^{2}\left\|P_{j} B\right\|^{2}<\infty \Rightarrow B: \mathscr{H} \rightarrow D(H)
$$


Hence, using the spectral theorem for $H$, we get, for any $\varphi$,

$$
\|H B \varphi\|^{2}=\sum_{j=1}^{\infty} \int_{\lambda}^{A} \lambda^{2} d\|E(\lambda) B \varphi\|^{2}
$$

where $[$ see $(2.2)]$

$$
\int_{\lambda}^{A} \lambda^{2} d\|E(\lambda) B \varphi\|^{2} \leqslant s^{2} \lambda_{j}^{2}\left\|P_{j} B\right\|^{2}\|\varphi\|^{2}
$$

which proves the boundedness of $H B$. Since the domain of $H$ is dense, $B^{*} H \subset(H B)^{*}$ is bounded as well.

There exists a basis of orthonormal eigenvectors $\left\{\varphi_{j}^{k}\right\}_{j \in N^{*}}^{k=1 \ldots m_{j}}$ of $H$ such that $P_{j}=\sum_{k=1}^{m_{j}}\left|\varphi_{j}^{k}\right\rangle\left\langle\varphi_{j}^{k}\right|, k=1, \ldots, m_{j}$, and $H \varphi_{j}^{k}=l_{j, k} \varphi_{j}^{k}$ with $\lambda_{j} \leqslant$ $l_{j, k} \leqslant \Lambda_{j}, k=1, \ldots, m_{j}$. Hence,

$$
\begin{aligned}
\|H B\|_{2}^{2} & =\left\|B^{*} H\right\|_{2}^{2} \\
& =\sum_{j=1}^{\infty} \sum_{k=1}^{m_{j}}\left\|B^{*} H \varphi_{j}^{k}\right\|^{2} \\
& \geqslant \sum_{j=1}^{\infty} \sum_{k=1}^{m_{j}}\left\|B^{*} \varphi_{j}^{k}\right\|^{2} \lambda_{j}^{2} \\
& =\sum_{j=1}^{\infty} \lambda_{j}^{2}\left\|B^{*} P_{j}\right\|_{2}^{2} \\
& \geqslant \sum_{j=1}^{\infty} \lambda_{j}^{2}\left\|B^{*} P_{j}\right\|^{2} \\
& =\sum_{j=1}^{\infty} \lambda_{j}^{2}\left\|P_{j} B\right\|^{2}
\end{aligned}
$$

with equalities everywhere if $m_{j}=1, \forall j \in N^{*}$.

If, furthermore, $\sup _{j \in \mathrm{N}^{*}} m_{j} \leqslant M$, then

$$
\begin{aligned}
\|H B\|_{2}^{2} & =\sum_{j=1}^{\infty} \sum_{k=1}^{m_{j}}\left\|B^{*} H \varphi_{j}^{k}\right\|^{2} \\
& \leqslant s^{2} \sum_{j=1}^{\infty} \sum_{k=1}^{m_{j}}\left\|B^{*} P_{j} \varphi_{j}^{k}\right\|^{2} \lambda_{j}^{2} \leqslant s^{2} M \sum_{j=1}^{\infty}\left\|B P_{j}\right\|^{2} \lambda_{j}^{2}
\end{aligned}
$$




\section{APPENDIX C. PROOF OF THEOREM 3.2}

We follow again the same steps as in the proof of Theorem 2.1 with $f(H)$ in place of $H$. The hypotheses on $a$ and the strict monotonicity of $f_{j}$ imply that $f_{j} \leqslant a^{2}\left(f_{j}\right)$ for $j \geqslant J, J$ large enough. Thus, for any $t$ [see (2.35)], if (i) holds,

$$
\begin{aligned}
& \sum_{j=J}^{\infty} f_{j}\left\|P_{j} R(t) \varphi\right\|^{2} \\
& \quad \leqslant\left\{\int_{0}^{t}\left(\sum_{j=J}^{\infty} a^{2}\left(f_{j}\right)\left\|P_{j} W^{o}(s)\right\|^{2}\right)^{1 / 2} d s\right\}^{2}\|\varphi\|^{2} \\
& \leqslant\left(\int_{0}^{t}\left\|W^{o}(s)\right\|_{2, a \cdot f(H)} d s\right)^{2}\|\varphi\|^{2}<\infty
\end{aligned}
$$

Hence, as above, we get that for any $t \geqslant 0, V(t): D\left(f^{1 / 2}(H)\right) \rightarrow D\left(f^{1 / 2}(H)\right)$, $R(t): \mathscr{H} \rightarrow D\left(f^{1 / 2}(H)\right)$, and thus $U(t): D\left(f^{1 / 2}(H)\right) \rightarrow D\left(f^{1 / 2}(H)\right)$. Then, under hypothesis (i), respectively (ii), using the monotonicity of $b$, we are led by arguments similar to $(2.43)$, respectively $(2.48)$, to minimize the quantity

$$
h(N, t)=f_{N}+r(t) f_{N+1} / a^{2}\left(f_{N+1}\right)
$$

for $N$ large enough, where

$$
r(t)=\left(\int_{0}^{1}\left\|W^{o}(s)\right\|_{2, a \cdot f(H)} d s\right)^{2}
$$

respectively

$$
r(t)=\left(\int_{0}^{t}|k(s)| d s\right)^{2}
$$

by a particular choice of $N(t)$. We do not attempt to take advantage of the presence of any decreasing function $g(N)$ in (C.2).

Lemma C.1. Let $h(N, t)$ be defined by (C.2), where $f$, respectively $a$, satisfies $\mathrm{H} 4$, respectively, H6. For each $t>0$, there exists an $N(t)$ such that

$$
h(N(t), t)=O\left(\left(a^{2}\right)^{-1}(r(t))\right)
$$

Remarks. If $r(t)$ is uniformly bounded in $t$, then $h\left(N_{0}, t\right)$ is, too, for any $N_{0}$ and the lemma is optimal in the same sense as Lemma 2.3. 
Proof. We can rewrite (C.2) as

$$
h(N, t)=f_{N}+r(t) / b\left(f_{N+1}\right)
$$

and we can assume that $r(t)$ tends to infinity as $t \rightarrow \infty$. Let $N(t)$ be the unique index such that

$$
f_{N(t)} \leqslant\left(a^{2}\right)^{-1}(r(t))<f_{N(t)+1}
$$

For $t$ large enough we can use the identity

$$
b\left(\left(a^{2}\right)^{-1}(x)\right)=x /\left(a^{2}\right)^{-1}(x) \quad \forall x \in\left[a^{2}(\beta), \infty[\right.
$$

and deduce from (C.7) that

$$
b\left(f_{N(t)+1}\right) \geqslant r(t) /\left(a^{2}\right)^{-1}(r(t))
$$

Hence

$$
h(N(t), t) \leqslant 2\left(a^{2}\right)^{-1}(r(t))=2 a^{-1}\left([r(t)]^{1 / 2}\right)
$$

This lemma ends the proof of the theorem.

\section{APPENDIX D. PROOF OF LEMMA 5.1}

According to the Schur condition (ref. 14, example 2.3, p. 143),

$$
\|G\| \leqslant \max \left(\sup _{j, r} \sum_{k=1}^{\infty} \sum_{s=1}^{m_{k}}\left|\left\langle\varphi_{j}^{r} \mid G \varphi_{k}^{s}\right\rangle\right|, \sup _{k, s} \sum_{j=1}^{\infty} \sum_{r=1}^{m_{j}}\left|\left\langle\varphi_{j}^{r} \mid G \varphi_{k}^{s}\right\rangle\right|\right)
$$

Thus it follows from our assumptions that we need to estimate

$$
\sup _{j^{\prime} \cdot r^{\prime}} \sum_{k=1}^{\infty} \sum_{s=1}^{m_{k}}\left|\left\langle\varphi_{j^{\prime}}^{r^{\prime}} \mid P_{j} W^{o}(t) \varphi_{k}^{*}\right\rangle\right| \leqslant \frac{C M}{j^{\gamma}} \sum_{\substack{k=1 \\ k \neq j}}^{\infty} \frac{1}{|k-j|^{p} k^{\gamma-\delta}}
$$

and

$$
\sup _{k, s} \sum_{j^{\prime}=1}^{\infty} \sum_{r^{\prime}=1}^{m_{j^{\prime}}}\left|\left\langle\varphi_{j^{\prime}}^{r^{\prime}} \mid P_{j} W^{o}(t) \varphi_{k}^{s}\right\rangle\right| \leqslant \frac{C M}{j^{\gamma-\delta}} \sup _{\substack{k \\ k \neq j}} \frac{1}{|k-j|^{p} k^{\gamma}}
$$

under the conditions $\gamma-\delta+p>1, \gamma>0$. It is readily seen that in any case the sup in (D.3) is bounded by $1 / j^{\min (p . \%)}$. The sum appearing in (D.2) is estimated using the integral test 


$$
\begin{array}{ll}
\sum_{k=a}^{b} f(k) \leqslant \int_{a}^{b+1} f(x) d x & \text { if } f(x) \text { is nondecreasing on }[a, b+1] \\
\sum_{k=a}^{b} f(k) \leqslant \int_{a-1}^{b} f(x) d x & \text { if } f(x) \text { is nonincreasing on }[a-1, b]
\end{array}
$$

on the function

$$
d(x)=\frac{1}{|x-j|^{p} x^{y^{\prime}}} \quad \text { for } \quad\left\{\begin{array}{l}
1 \leqslant x \leqslant j-1 \\
x \geqslant j+1
\end{array}\right.
$$

where $\gamma^{\prime}=z-\delta$. The resulting integrals are then analyzed by means of the technique described in the appendix of ref. 9. In (D.2), however, we have to accommodate the term $|k-j|^{-p}$, which may be singular as $k \simeq j$ and was absent in ref. 9. The analysis is nevertheless quite similar, so we only give the main steps (we will denote by the same symbol $c$ all constants independent of $j$ in what follows).

Let $D_{d}=\{x \in \mathbf{R} \mid 1 \leqslant x \leqslant j-1$ or $x \geqslant j+1\}$ and $x_{0}$ be such that $d^{\prime}\left(x_{0}\right)=0$. We need to consider four cases:

1. $p>0$ and $\gamma^{\prime}>0 \Rightarrow x_{0}<j-1$, for $j$ large enough.

2. $p>1$ and $\gamma^{\prime} \leqslant 0 \Rightarrow x_{0} \notin D_{d}$.

3. $p<0$ and $\gamma^{\prime}>1 \Rightarrow x_{0}>j+1$, for $j$ large enough.

4. $p=0$ and $\gamma^{\prime}>1 \Rightarrow \nexists$ such $x_{0}$.

For case 4 we get

$$
\sum_{\substack{k=1 \\ k \neq j}}^{\infty} d(k) \leqslant \sum_{k=1}^{\infty} d(k) \leqslant d(1)+\int_{1}^{\infty} d(x) d x<c
$$

For case 2, similarly, and asymptotically as $j \rightarrow \infty$,

$$
\begin{aligned}
& \sum_{k=1}^{j-1} d(k) \leqslant d(j-1)+\int_{1}^{j-1} d(x) d x \leqslant c / j^{\gamma^{\prime}}+\int_{1}^{j-1} d(x) d x \\
& \sum_{k=j+1}^{\infty} d(k) \leqslant d(j+1)+\int_{j+1}^{\infty} d(x) d x \leqslant c / j^{\prime}+\int_{j+1}^{\infty} d(x) d x
\end{aligned}
$$

In case 1 we proceed as follows for $\sum_{k=1}^{j-1} d(k)$ :

$$
\begin{aligned}
\sum_{k=1}^{j-1} d(k) & =d(1)+\sum_{k=2}^{\left[. x_{0}\right]} d(k)+\sum_{k=\left[x_{0}\right]+1}^{j-2} d(k)+d(j-1) \\
& \leqslant c / j^{p}+\int_{1}^{j-1} d(x) d x+c / j^{\gamma^{\prime}}
\end{aligned}
$$


whereas (D.8) holds for $\sum_{k=j+1}^{\infty} d(k)$. Finally, for case 3 we get by similar manipulations

$$
\begin{aligned}
\sum_{k=1}^{j-1} d(k) & \leqslant c / j^{p}+\int_{1}^{j-1} d(x) d x \\
& \leqslant c / j^{\prime}+\int_{1}^{j-1} d(x) d x \\
\sum_{k=j+1}^{\infty} d(k) & \leqslant 2 d\left(\left[x_{0}\right]\right)+\int_{j+1}^{\infty} d(x) d x \\
& \leqslant c / j^{p+y^{\prime}}+\int_{j+1}^{\infty} d(x) d x
\end{aligned}
$$

using $x_{0}=\gamma^{\prime} j /\left(p+\gamma^{\prime}\right)$. It remains to estimate the integrals. Using the techniques of ref. 9 , we get

$$
\int_{j+1}^{\infty} d(x) d x \leqslant \begin{cases}c / j^{p+\gamma^{\prime-1}} & \text { if } p<1 \\ c \ln j / j^{\prime} & \text { if } p=1 \\ c / j^{\gamma^{\prime}} & \text { if } p>1\end{cases}
$$

On the other hand,

$$
\int_{1}^{j-1} d(x) d x=\int_{1}^{j / 2} d(x) d x+\int_{j / 2}^{j-1} d(x) d x \equiv I_{1}+I_{2}
$$

where

$I_{1} \leqslant\left\{\begin{array}{ll}c / j^{p+\gamma^{\prime-1}} & \text { if } \gamma^{\prime}<1 \\ c \ln j / j^{p} & \text { if } \gamma^{\prime}=1 \\ c / j^{p} & \text { if } \gamma^{\prime}>1\end{array} \quad\right.$ and $\quad I_{2} \leqslant \begin{cases}c / j^{p+\gamma^{\prime}-1} & \text { if } p<1 \\ c \ln j / j^{\prime} & \text { if } p=1 \\ c / j^{\gamma^{\prime}} & \text { if } p>1\end{cases}$

Gathering these estimates in (D.1), we finally get the result.

\section{ACKNOWLEDGMENTS}

I wish to thank G. Hagedorn, J. Howland, and G. Karner for very helpful and friendly discussions. Financial support from Fonds National Suisse de la Recherche Scientifique, Grant 8220-037200, and the hospitality of the Mathematics Department of Virginia Tech are acknowledged. 


\section{REFERENCES}

1. L. Bunimovich, H. R. Jauslin, J. L. Lebowitz, A. Pellegrinotti, and P. Nielaba, Diffusive energy growth in classical and quantum driven oscillators, J. Stat. Phys. 62:793-817 (1991).

2. J. M. Combes, Connections between quantum dynamics and spectral properties of timeevolution operators, in Differential Equations with Applications to Mathematical Physics, W. F. Ames, E. M. Harrell, and J. V. Herod, eds. (Academic Press, Boston, 1993).

3. P. Duclos and P. Stovicek, Floquet Hamiltonians with pure point spectrum, CPT94/P.3128 preprint (1994).

4. P. Duclos and P. Stovicek, Quantum Fermi accelerators with pure-point quasispectrum, CPT-94/P.3127 preprint (1994).

5. V. Enss and $\mathrm{K}$. Veselić, Bound states and propagating states for time-dependent Hamiltonians, Ann. Inst. H. Poincaré A 39:159-191 (1983).

6. I. Guarneri and G. Mantica, On the asymptotic properties of quantum dynamics in the presence of a fractal spectrum, Ann. Inst. H. Poincaré A 61:369-379 (1994).

7. G. Hagedorn, M. Loss, and J. Slawny, Nonstochasticity of time-dependent quadratic Hamiltonians and spectra of canonical transformations, J. Phys. A 19:521-531 (1986).

8. J. Howland, Floquet operator with singular spectrum I, Ann. Inst. H. Poincaré $A$ 49:309-323 (1989).

9. J. Howland, Floquet operator with singular spectrum II, Ann. Inst. H. Poincaré A 49:325-334 (1989).

10. J. Howland, Floquet operator with singular spectrum III, preprint (1995).

11. H. R. Jauslin, Stability and chaos in classical and quantum Hamiltonian systems, in II Granada Seminar on Computational Physics, P. Garrido and J. Marro, eds. (World Scientific, Singapore, 1983).

12. A. Joye. Absence of absolutely continuous spectrum of Floquet operators, J. Stat. Phys. 75:929-952 (1994).

13. H. R. Jauslin and J. L. Lebowitz, Spectral and stability aspects of quantum chaos, Chaos 1:114-137 (1991).

14. T. Kato, Perturbation Theory for Linear Operators (Springer-Verlag, Berlin, 1966).

15. S. G. Krein, Linear Differential Equations in Banach Spaces (American Mathematical Society, Providence, Rhode Island, 1971).

16. Y. Last, Quantum dynamics and decompositions of singular continuous spectra, preprint (1995).

17. G. Nenciu, Floquet operators without absolutely continuous spectrum, Ann. Inst. H. Poincaré A 59:91-97 (1993).

18. G. Nenciu, Adiabatic theory: Stability of systems with increasing gaps, CPT-95/P.3171 preprint (1995).

19. C. R. de Oliveira, Some remarks concerning stability for nonstationary quantum systems, J. Stat. Phys. 78:1055-1066 (1995). 